LETTER TO THE EDITOR

\title{
Quantum Turbulence
}

\section{Kostas Davanas}

Ministry of Transport, Saint Stephen, Greece 053aib@otenet.gr

DOI: $10.31275 / 20201825$

Creative Commons: CC-BY-NC

Last year, in an article in the JSE, Lear (2019) elaborated on the debate/ literature about the ongoing mysteries of non-locality and causality in the quantum world by addressing, in a novel way (among other issues), the "classical" enigma of particle entanglement and "spooky action at a distance" via the so-called collapse of the wave-function. Invoking the collapse of the wave-function brings up the role of measurement in the process, a matter that herein is further elaborated/addressed from an alternative (than it appears in the literature) point of view since it is potentially of significant importance when trying to explore the mysteries of the quanta.

In line with the wide-spectrum nature/audience and interdisciplinary approach of the JSE, the work by Felder (1999) will be followed because, albeit simplified (e.g., in the eyes of a professional physicist), it contains the necessary mathematics, at a non-advanced level, to be accessible to a wide readership both from a qualitative ("popular science") and a quantitative ("equations-having") point of view.

Thus, following Felder(1999), one sees that when pairs of entangled particles are shot in opposite directions and then a (binary) property of theirs is measured by trimodal detectors (i.e. measuring devices independently and continuously and randomly being set at any one of only three configurations) that are positioned very far apart from each other (so that the entangled particles cannot communicate without exceeding the speed of light, i.e. an "impossible" affair), the detectors' measurement streams (Aspect et al., 1982a, 1982b) do not (statistically) 
correspond to each other at least in 5/9ths of the time (as expected by classical reckoning, i.e. Bell's [1964] inequality), but instead correspond only $50 \%$ of the time (as calculated by the "exotic" equations of quantum mechanics which allow for instant communication between any two points in the universe via the instantaneous collapse of the wave-function throughout the cosmos). This so-called "non-locality," or as Lear (2019) prefers it "nonlocal causality," implies that each detector's measurement traverses instantly the entire world (how this may be done constitutes a deep mystery).

To make a measurement, a detector must somehow interact with a target (e.g., one of the particles of an entangled pair) even if there is "nothing" in between them. But according to state-of-the-art physics, it does seem that there is a very good chance that there is no such thing as nothingness or absolute void or emptiness and the like (Laughlin, 2005; Silk, 2005; Yiu, 2017; Koga \& Hayakawa, 2017). Thus, in spite of the "disproof" of the ether back in the 19th century, and if the word "medium" would better be avoided due to its negative connotations (vis-à-vis the ether), it has still to be admitted (referring to the quantum realm) that during the interaction between detector and target the "fabric" of the involved (in the interaction) micro-cosmos (whether it be the unremittingly fluctuating quantum vacuum or whatever else) might, or most probably shall, get perturbed-and this might, as well, affect any subsequent measurements (and/or targets) if the "fabric" (as "middleman") is not allowed to "relax." If that is indeed true, then via a detector's continuous random trimodal changeovers, in the aforedefined "fabric" (or, more accurately, in the detector-target-"fabric" ensemble), either some kind of pattern will be established or some sort of "turbulence" will set in. In the latter case, the obvious outcome of any (statistical) examinations/comparisons of the data streams of the two far-away detectors will be a 50\% correspondence as actually measured experimentally (nothing spooky here, it is like statistically examining/comparing the toss sequences/streams of two coins at two different sites no matter if they are separated by meters or parsecs); in the former case, descriptive equations must (at least in principle) be possible to be derived, which upon solution shall give the probability of correspondence of the data streams of the two detectors.

Such a rationale can actually be employed to the well-known 
"double-slit experiment" (e.g. Al-Khalili, 2013) as well: When there is no detector to count how many particles go through one of the slits, an interference pattern develops; otherwise, perhaps due to the perturbation of the (afore-defined) "fabric," when a counting detector is functioning, a two-zone pattern appears.

Therefore, a more detailed look at the (quantum or otherwise) vacuum (alias "fabric") and its role as the "middleman" of interactions may be in order; at least to tackle any relevant "loopholes" in Bell (1964) test experiments and (any) ambiguities about "hidden variables" of entangled particles (Einstein et al., 1935); moreover, to probe whether this detector-target-via- "fabric" interaction inflicts any kind of alteration (reversible or irreversible) on the target itself.

\section{REFERENCES}

Al-Khalili, J. (2013). The double slit experiment—Royal Institution video. www.youtube.com/watch?v=AgtKncAdlHQ

Aspect, A., Grangier, P., \& Roger, G. (1982a, July 12). Experimental realization of Einstein-Podolsky-Rosen-Bohm gedankenexperiment: A new violation of Bell's inequalities. Physical Review Letters, 49(2), 91-94. https://doi.org/10.1103/PhysRevLett.49.91

Aspect, A., Dalibard, J., \& Roger, G. (1982b, December 20). Experimental test of Bell's inequalities using time-varying analyzers. Physical Review Letters, 49(25), 1804-1807. https://doi.org/10.1103/PhysRevLett.49.1804

Bell, J. S. (1964). On the Einstein Podolsky Rosen paradox. Physics, 1(3), 195-200. https://doi.org/10.1103/PhysicsPhysiqueFizika.1.195

Einstein, A., Podolsky, B., \& Rosen, N. (1935, May 15). Can quantum-mechanical description of physical reality be considered complete? Physical Review, 47(10), 777-780. https://journals.aps.org/pr/abstract/10.1103/PhysRev.47.777

Felder, G. (1999). Spooky action at a distance-An explanation of Bell's theorem. www.felderbooks.com/papers/bell.html

Koga, J. K., \& Hayakawa, T. (2017, May 17). Possible precise measurement of Delbrük scattering using polarized photon beams. Physical Review Letters, 118, 204801. https://doi.org/10.1103/PhysRevLett.118.204801

Laughlin, R. B. (2005). A different universe: Reinventing physics from the bottom down. Basic Books.

Lear, C. W. (2019, March). The case for advance wave causality. Journal of Scientific Exploration, 33(1), 61-72. https://doi.org/10.31275/2019.1232

Silk, J. (2005). On the shores of the unknown: A short history of the universe. Cambridge University Press.

Yiu, Y. (2017). A study about nothing. www.insidescience.org/news/study-about-nothing 